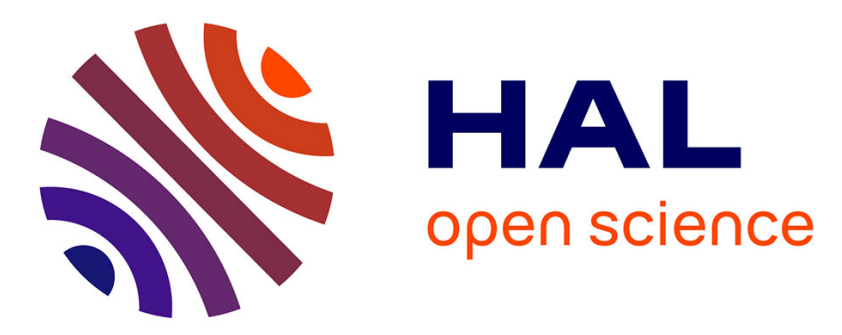

\title{
Détermination du travail de sortie par comparaison des distributions énergétiques des ions secondaires
}

\author{
Marc Bernheim
}

\section{To cite this version:}

Marc Bernheim. Détermination du travail de sortie par comparaison des distributions énergétiques des ions secondaires. Revue de Physique Appliquée, 1989, 24 (5), pp.515-527. 10.1051/rphysap:01989002405051500 . jpa-00246077

\section{HAL Id: jpa-00246077 https://hal.science/jpa-00246077}

Submitted on 1 Jan 1989

HAL is a multi-disciplinary open access archive for the deposit and dissemination of scientific research documents, whether they are published or not. The documents may come from teaching and research institutions in France or abroad, or from public or private research centers.
L'archive ouverte pluridisciplinaire HAL, est destinée au dépôt et à la diffusion de documents scientifiques de niveau recherche, publiés ou non, émanant des établissements d'enseignement et de recherche français ou étrangers, des laboratoires publics ou privés. 
Classification

Physics Abstracts

$79.20 \mathrm{~N}-07.80$

\title{
Détermination du travail de sortie par comparaison des distributions énergétiques des ions secondaires
}

\author{
Marc Bernheim \\ Laboratoire de Physique des Solides associé au CNRS (LA 002), Université Paris-Sud, Bât. 510, 91405 Orsay, \\ France
}

(Reçu le 17 novembre 1988, révisé le 10 janvier 1989, accepté le 31 janvier 1989)

\begin{abstract}
Résumé. - L'accroissement des rendements d'émission ionique dans des réactions chimiques superficielles s'accompagne souvent de modifications des distributions énergétiques des ions secondaires. Mais les exemples rapportés ici montrent que, lorsque la collecte des ions secondaires est assurée par des optiques électrostatiques à immersion, la forme des distributions des ions de très basse énergie initiale est conservée. Les seules variations observées se limitent alors à des décalages qui résultent directement des variations de travail de sortie. Ainsi, au cours d'une fixation de césium par exemple, la comparaison des positions des distributions énergétiques successives des ions négatifs permet une détermination des variations de travail de sortie à mieux que $0,1 \mathrm{eV}$ près, précision bien supérieure à celle que laisserait supposer la seule bande passante du spectromètre. Un réglage instrumental est proposé pour faciliter des comparaisons très précises des distributions énergétiques d'espèces ioniques différentes.
\end{abstract}

\begin{abstract}
The increase of the secondary ion yields during superficial chemical reactions is often followed by modifications of the ion energy distributions. However, in most cases, when immersion electrostatic lenses are used to collect the secondary ions, the shape of distribution curves stays nearly constant for the ions ejected with very low initial energies. Therefore the only measured variations are shifts directly related to the change of the sample work function. Different experimental situations, related to the cesium adsorption, establish the accuracy of this work function determination : precision better than $0.1 \mathrm{eV}$ can easily be reached using an energy dispersive spectrometer. An experimental method is proposed to extend this accurate comparison to the energy distributions of different ion species.
\end{abstract}

\section{Introduction.}

On sait que l'émission d'ions secondaires par des cibles solides bombardées par un faisceau d'ions primaires est fortement influencée par la composition chimique des échantillons. Souvent la fixation d'atomes de césium, d'oxygène ou d'autres éléments chimiquement actifs est utilisée pour accroître les rendements ioniques et permettre par là des analyses très sensibles.

La plupart du temps, ces réactions superficielles conduisent à la formation de couches dipolaires qui modifient le travail de sortie de la surface examinée. Or le travail de sortie influence les échanges électroniques entre les particules éjectées et la surface, il constitue donc l'un des paramètres qui contrôlent l'ionisation et intervient de ce fait dans de nombreuses expressions théoriques [1]. Ces mêmes raisons rendent souvent nécessaire une mesure du travail de sortie dans les études expérimentales des modifica- tions des processus d'ionisation par des réactions chimiques superficielles.

La méthode proposée ici repose sur la mesure du décalage des distributions énergétiques. En effet, toute variation du travail de sortie modifie le potentiel de la surface de l'échantillon et entraîne une variation identique de la différence de potentiel de contact entre la surface étudiée et une électrode de référence. La mesure des décalages des distributions énergétiques qui en résultent conduit donc à une détermination directe des variations de travail de sortie [2]. Le principal intérêt de ce procédé est de permettre une détermination des travaux de sortie dans les conditions même des expériences d'émission ionique secondaire, sans aucune adaptation instrumentale.

Mais toutefois, dans de tels examens, on peut craindre que la modification progressive des processus d'ionisation ne s'accompagne aussi de changements de forme des distributions énergétiques. La 
mesure des translations d'ensemble liées à la variation de potentiel de contact se trouverait ainsi perturbée. En particulier, la détermination du zéro de la distribution deviendrait un point critique susceptible de remettre en cause la validité de la méthode expérimentale, comme l'a suggéré $\mathrm{K}$. Wittmaack [3]. Pour apporter une réponse à cette question, nous avons examiné différentes situations expérimentales et mis au point une méthode qui permet une comparaison très précise des distributions énergétiques d'espèces ioniques différentes.

\section{Quelques résultats expérimentaux.}

On sait que de très forts abaissements du travail de sortie sont produits par l'adsorption de césium [4]. Des variations tout à fait similaires sont relevées sur les surfaces bombardées par des ions de gaz rare et alimentées simultanément par un jet moléculaire de vapeur de césium $[5,6]$. Dans ces dernières conditions, la concentration superficielle en césium est le résultat d'un équilibre dynamique entre la fixation du césium sur la surface et l'éjection par la pulvérisation cathodique des atomes chimisorbés; ainsi la composition superficielle peut être ajustée librement par le contrôle du flux de césium incident.

1) Sur la figure 1 , nous avons reporté quelques enregistrements du début des distributions énergétiques des ions $\mathrm{Cu}^{-}$relevés sur un échantillon monocristallin de cuivre pur pour des concentrations superficielles en césium croissantes. Pour faciliter la comparaison des formes des différentes distributions reportées sur cette figure, nous avons opéré une normalisation au niveau des maxima qui compense les grandes variations du rendement ionique $\mathrm{Cu}^{-}$ induites par l'adsorption de césium : l'augmentation, sur plus de 5 décades de l'intensité des ions $\mathrm{Cu}^{-}$n'entraîne guère de modification de la forme des distributions énergétiques (si l'on excepte les très légères variations au niveau des premiers enregistrements).

Les décalages des distributions énergétiques observés (Fig. 1) reproduisent alors les changements de potentiel de contact entre la surface de l'échantillon et une référence au niveau du spectromètre. Il suffit de relever la translation de l'ensemble des distributions pour obtenir une mesure in situ des variations de travail de sortie liées à la fixation du césium à la surface de l'échantillon. Ces décalages peuvent en principe être repérés à un niveau relatif quelconque de ces courbes, mais la forme des distributions est telle qu'une détermination aux faibles énergies initiales est bien plus précise puisqu'un léger déplacement se traduit par une forte variation de l'intensité transmise.

2) Comme les rendements d'émission des espèces ioniques émises par un même échantillon peuvent être influencés différemment par la fixation du

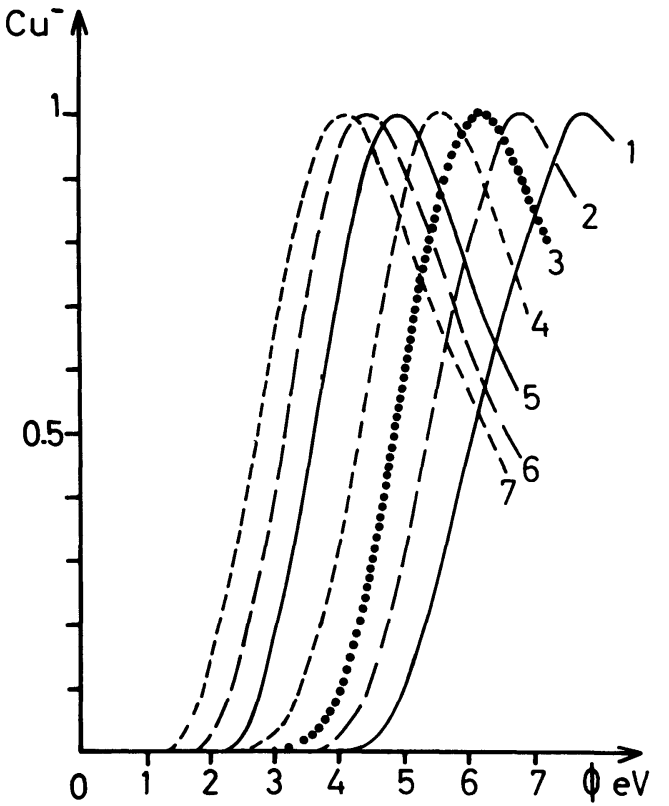

Fig. 1. - Influence d'une fixation de césium sur le début des distributions énergétiques des ions $\mathrm{Cu}^{-}$mesurés sur un monocristal de cuivre pur non orienté. Différents facteurs multiplicatifs $\left(1 ; 2,5 \times 10^{-2} ; 5,1 \times 10^{-3} ; 1,1 \times 10^{-3}\right.$; $1,9 \times 10^{-4} ; 1,6 \times 10^{-5} ; 3 \times 10^{-6}$ ) ont été appliqués aux courbes $1,2, \ldots$ et 7 pour prendre en compte l'accroissement des probabilités d'ionisation par une fixation croissante de césium. Le minimum du travail de sortie est enregistré pour la courbe 7 ; il correspond à une concentration superficielle en césium égale à 0,16 at. Cs/at. $\mathrm{Cu}$, valeur déterminée par l'examen des ions primaires $\mathrm{Ne}^{+}$rétrodiffusés [5]. Pour les relevés ci-dessus, la bande passante du spectromètre vaut $2,4 \mathrm{eV}$; le diaphragme de contraste fixe à $0,3 \mathrm{eV}$ la limite supérieure de l'énergie cinétique transversale des ions (voir paragraphe 4). L'angle d'incidence du faisceau primaire, constitué d'ions $\mathrm{Ar}^{+}$de $14 \mathrm{keV}$, est voisin de $45^{\circ}$; la plage érodée déborde largement de part et d'autre de la surface alimentée en césium et du champ où s'effectue la collecte des ions secondaires. La pression résiduelle dans l'enceinte $5 \times 10^{-9}$ torr est abaissé au niveau d'un ultra-vide par un écran cryogénique à $15 \mathrm{~K}$ entourant l'échantillon.

[Recordings of $\mathrm{Cu}^{-}$ion distributions measured on a copper crystal for increasing cesium coverages. Scaling factors $1 ; 2.5 \times 10^{-2} ; 5.1 \times 10^{-3} ; 1.1 \times 10^{-3}$; $1.9 \times 10^{-4} ; 1.6 \times 10^{-5} ; 3 \times 10^{-6}$ have been applied to curves $1,2 \ldots 7$ in order to take into account the increase of ionisation probabilities. During these recordings the spectrometer energy band pass was fixed at $2.4 \mathrm{eV}$.]

césium, il est important de vérifier que la mesure des variations de travail de sortie reste indépendante de l'espèce ionique isolée. Sur la figure 2 on a reporté à titre d'exemple les décalages des distributions des ions $\mathrm{Cu}^{-}$et $\mathrm{Cl}^{-}$relevés sur un même échantillon de cuivre pur en fonction de la température du four qui contrôle le flux incident de vapeur de césium. Dans ces mesures, l'intensité des ions $\mathrm{Cl}^{-}$est accrue par une adsorption sur la surface de l'échantillon de molécules de fréon 12 introduites à très basse 


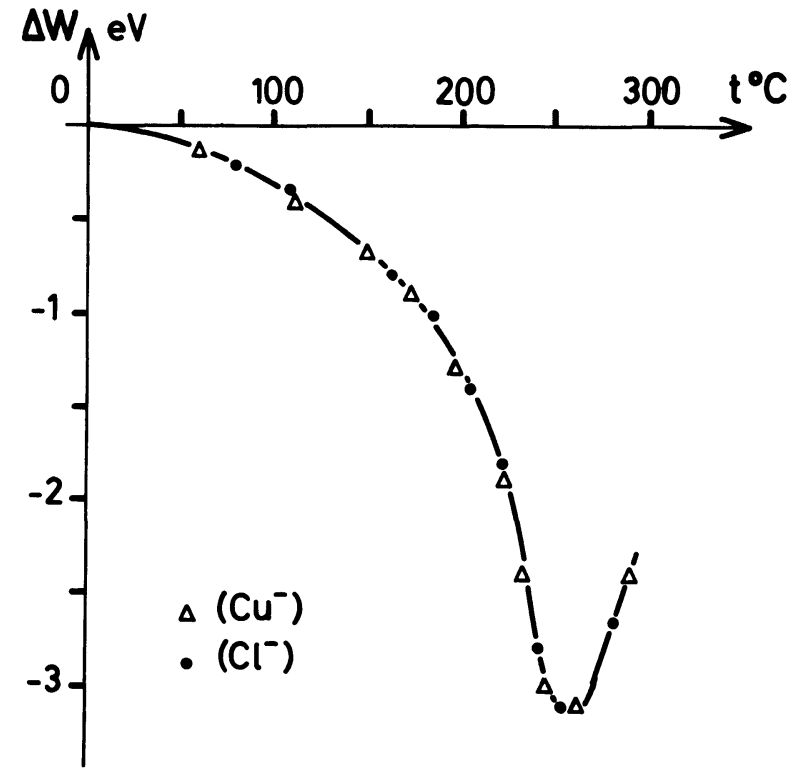

Fig. 2. - Comparaison des variations de travail de sortie déduites des distributions des ions $\mathrm{Cu}^{-}$et $\mathrm{Cl}^{-}$relevées sur un même cristal de cuivre pur. Le paramètre porté en abscisse est la température du four qui produit le jet moléculaire alimentant la surface en césium. Les conditions expérimentales sont identiques à celles des enregistrements de la figure 1.

[Comparison of work function variations deduced from the shifts of $\mathrm{Cu}^{-}$and $\mathrm{Cl}^{-}$energy distributions measured on a copper crystal. On the $x$ axis, the temperature of the liquid cesium reservoir providing the neutral cesium flux is reported.]

pression dans l'enceinte. L'affinité électronique du chlore, particulièrement élevée $(3,62 \mathrm{eV})$, conduit à une ionisation quasi totale de cet élément quelle que soit la concentration superficielle en césium ; l'intensité des ions $\mathrm{Cl}^{-}$ne varie donc que très légèrement durant la fixation du césium à part une baisse d'un facteur 2 environ attribuée à une légère décroissance des rendements de pulvérisation. Les résultats, reportés figure 2 montrent le très bon accord entre ces séries de mesures. Les grandes différences de variation des rendements ioniques au cours de la fixation de césium s'accompagnent ainsi de décalages quasi identiques des distributions des ions $\mathrm{Cu}^{-}$et $\mathrm{Cl}^{-}$.

3) La mesure de telles variations de travail de sortie pourrait aussi reposer sur la comparaison des distributions énergétiques des ions positifs formés à partir des constituants majeurs ou des impuretés de l'échantillon. Mais souvent, une formation additionnelle d'ions dans des processus d'ionisation retardée [7] perturbe la détermination des décalages.

Toutefois pour les ions secondaires formés à partir des atomes d'argon du faisceau d'ions primaires, ce processus d'ionisation retardée devient dominant. Ces ions résultent d'éléments primaires implantés à
$14 \mathrm{keV}$ sous incidence de $45^{\circ}$ environ. Ces atomes sont désorbés avec des énergies initiales quasi nulles lorsque la pulvérisation les place à proximité de la surface, et peuvent être ensuite ionisés par transfert de charge $\left(^{1}\right)$ avec le faisceau d'ions incident à plus ou moins grande distance de la surface ; comme le champ électrostatique accélérateur n'agit que sur une portion de la trajectoire, l'énergie cinétique de ces ions à la sortie de l'optique collectrice reste toujours inférieure à celle des ions secondaires; les distributions énergétiques présentent alors un aspect inversé avec une croissance progressive suivie d'une chute rapide lorsque l'ionisation se produit au niveau de la surface.

La figure 3 résume, dans le cas de l'argon, les modifications de distribution des ions $\mathrm{A}^{+}$provoquées par une fixation croissante de césium sur un monocristal de cuivre. Le décalage des distributions énergétiques peut être facilement déterminé au

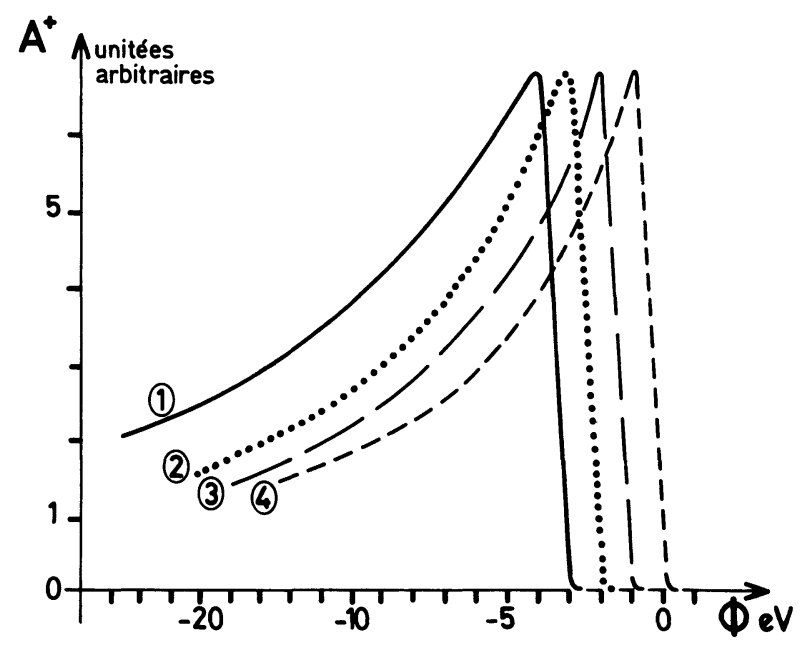

Fig. 3. - Décalages des distributions énergétiques des ions argon au cours de l'adsorption de césium sur du cuivre pur. La polarisation de l'échantillon a été réduite à $500 \mathrm{~V}$ pour permettre un bon centrage de la plage bombardée sur le champ imagé. L'énergie des ions primaires au niveau de l'échantillon vaut alors $9,5 \mathrm{keV}$; la bande passante du spectromètre s'élève à $0,8 \mathrm{eV}(\ell=1 \mathrm{~mm}$, voir paragraphe 3$)$. Des conditions expérimentales analogues permettent une calibration de la couverture de la surface en césium à partir de la rétrodiffusion à $135^{\circ}$ d'un faisceau d'ions primaires ${ }^{20} \mathrm{Ne}^{+}$[5]. Au niveau du minimum de travail de sortie (courbe 4), la couverture atteint 0,16 at. $\mathrm{Cs} / \mathrm{at}$. $\mathrm{Cu}$, valeur comparable à celle mesurée sur des surfaces non soumises à la pulvérisation.

[Shifts of $\mathrm{Ar}^{+}$ion energy distributions curves 1,2, 3, 4 measured on copper for increasing superficial cesium concentrations.]

(1) Pour d'autres éléments, on peut observer une ionisation retardée spontanée; l'intensité des ions ainsi formés ne varie plus de façon quadratique avec la densité du faisceau primaire [7]. 
niveau de la chute. Ainsi, d'après ces relevés, le travail de sortie diminue progressivement, passe par un minimum $(\Delta W=-3,1 \mathrm{eV})$, puis croît légèrement pour se stabiliser à une valeur $(\Delta W=-2,5 \mathrm{eV})$ indépendante du flux incident de césium. Ces variations sont en accord avec les relevés déduits des distributions des ions $\mathrm{Cu}^{-}$et $\mathrm{Cl}^{-}$.

4) Le problème pratique posé par la mesure du travail de sortie apparaît plus directement sur les enregistrements reportés sur les figures $4 a$ et $5 \mathrm{a}$ relatifs aux ions $\mathrm{Cu}^{-}$et $\mathrm{Si}^{-}$mesurés respectivement sur des monocristaux d'oxyde cuivreux et de silicium pur. Ici, en l'absence de toute couverture de césium, l'émission est déjà bien plus intense $\left(10^{4}\right.$ et $10^{5}$ coups par seconde) que celle mesurée dans les mêmes conditions sur le cuivre pur $\left({ }^{2}\right)$; et si l'adsorption de césium s'accompagne toujours d'un renforcement considérable des intensités secondaires, cette fois la forme des distributions énergétiques change nettement. Un processus d'ionisation, lié à la présence de césium, se met progressivement en place avant de devenir dominant. Les translations induites par le changement de travail de sortie ne peuvent plus être mesurées sans précautions puisque le relevé dépend cette fois du niveau relatif $h$ où il est effectué (Figs. 4b et 5b).

Toutefois, les mesures de variation de travail de sortie sont peu altérées tant que ce niveau est limité à 5 ou $10 \%$; de plus, l'extrapolation linéaire des courbes des figures $4 \mathrm{~b}$ et $5 \mathrm{~b}$ vers les valeurs inférieures de $h$ ne laisse pas prévoir de comportement singulier.

La situation de la figure 6 pourrait correspondre au cas extrême d'une coexistence de deux processus d'ionisation contribuant à des distributions énergétiques partiellement séparées ( ${ }^{3}$ ). Dans ce cas, pour effectuer la détermination des variations du travail de sortie, il sera nécessaire d'isoler de préférence une autre espèce ionique dont la distribution énergétique et la probabilité d'ionisation varieraient moins avec la composition superficielle; il existe souvent $\mathrm{du}$ chlore à une concentration résiduelle suffisante pour permettre ces relevés à partir des ions $\mathrm{Cl}^{-}$.

\section{Quelques indications sur le montage expérimental.}

Avant de développer les problèmes pratiques posés par la détermination du travail de sortie rappelons les principales caractéristiques du microscope ionique utilisé.

(2) L'émission d'ions $\mathrm{Cu}^{-}$reste inférieure à $100 \mathrm{cps}$ sur le cuivre pur, exempt d'oxygène et en l'absence de toute fixation d'atomes alcalins.

$\left({ }^{3}\right)$ Expérimentalement, une telle situation n'a jamais été mise en évidence pour les ions d'énergie initiale inférieure à $10 \mathrm{eV}$, elle a été remarquée pour les ions émis à plus hautes énergies initiales [13].

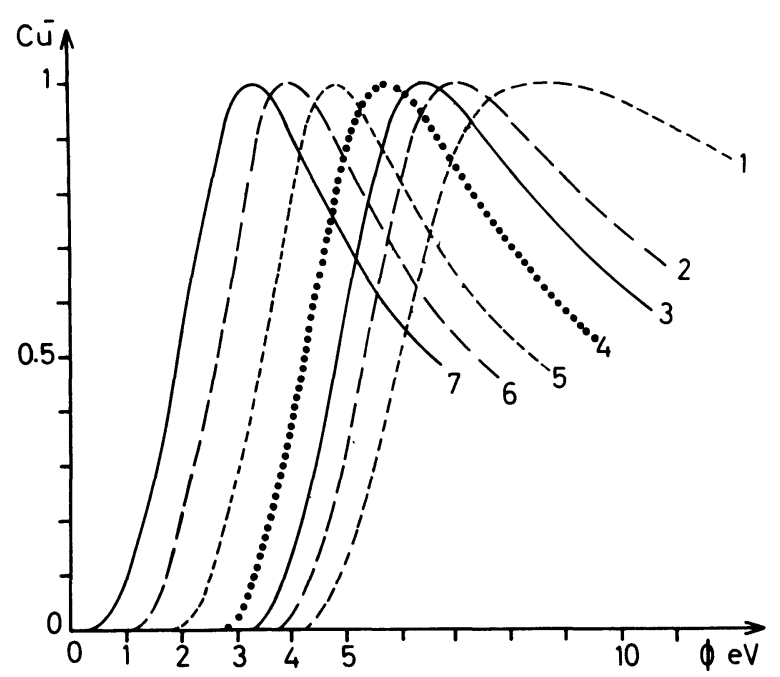

a)

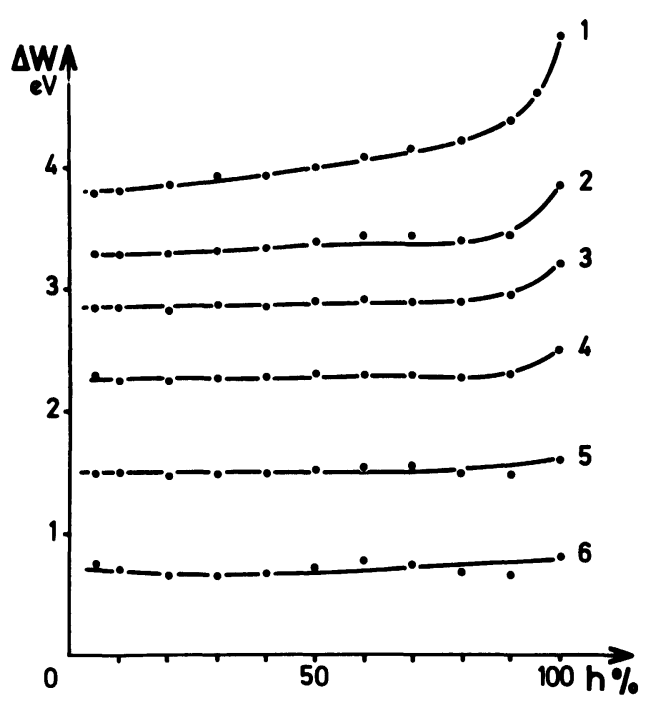

b)

Fig. 4. - a) Enregistrements du début des distributions énergétiques des ions $\mathrm{Cu}^{-}$mesurées sur un cristal d'oxyde cuivreux pour des concentrations superficielles en césium croissantes ; les conditions expérimentales sont identiques à celles de la figure 1 . Les facteurs multiplicatifs : 1 ; $2 \times 10^{-2} ; 7,6 \times 10^{-3} ; 3,3 \times 10^{-3} ; 2,1 \times 10^{-3} ; 1,6 \times 10^{-3}$ et $1,15 \times 10^{-3}$ ont été appliqués aux courbes $1,2 \ldots 7$ pour prendre en compte l'accroissement des probabilités d'ionisation par la fixation du césium. b) Mesures des variations de travail de sortie déduites des enregistrements de la figure $4 \mathrm{a}$. Les translations des distributions énergétiques déterminées par référence à la courbe 7 dépendent légèrement de la hauteur relative $h \%$ considérée.

[a) Recordings similar to those in figure 1 , measured on a $\mathrm{Cu}_{2} \mathrm{O}$ crystal. Scaling factors $1 ; 2 \times 10^{-2} ; 7.6 \times 10^{-3}$; $3.3 \times 10^{-3} ; 2.1 \times 10^{-3} ; 1.6 \times 10^{-3} ; 1.15 \times 10^{-3}$ have been applied to curves $1 . .7$ in order to compensate for the increase of ionisation probabilites. b) Shifts of energy distributions measured at several relative levels in figure 4a.]

Les ions secondaires émis par l'échantillon sont collectés par un objectif électrostatique à immer- 


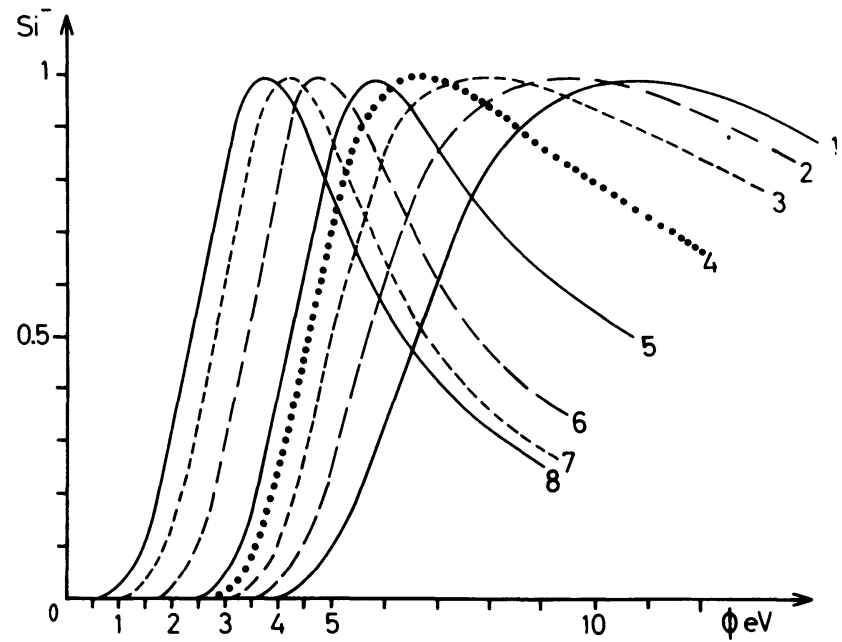

a)

Fig. 5. - a) Enregistrements du début des distributions énergétiques des ions $\mathrm{Si}^{-}$émis par un cristal de silicium pour des concentrations superficielles en césium croissantes ; les conditions expérimentales sont identiques à celles de la figure 1 . Différents facteurs multiplicatifs ( 1 ; $1,7 \times 10^{-1} ; 4,6 \times 10^{-2} ; 1,12 \times 10^{-2} ; 1,8 \times 10^{-3}$; $8,9 \times 10^{-5}$ et $2,5 \times 10^{-5} ; 1,5 \times 10^{-5}$ ) ont été appliqués aux courbes $1,2, \ldots 8$ pour prendre en compte l'accroissement des probabilités d'ionisation qui résultent de la fixation du césium. b) Mesures des variations de travail de sortie déduites des enregistrements de la figure 5a. Les translations ont été mesurées par référence à la courbe 8 . Aux faibles couvertures en césium, la mesure dépend du niveau relatif $h$ choisi pour la mesure. Les écarts se réduisent à $\pm 0,1 \mathrm{eV}$ si la hauteur relative reste inférieure à $15 \%$.

[a) Recording of $\mathrm{Si}^{-}$ion distributions for increasing cesium coverage. Scaling factors $1 ; 1.7 \times 10^{-1}$; $4.6 \times 10^{-2} ; 1.12 \times 10^{-2} ; 1.8 \times 10^{-3} ; 8.9 \times 10^{-5} ;$ $2.5 \times 10^{-5} ; 1.5 \times 10^{-5}$ have been applied to compensate for the increase of ionization probabilities. b) Shifts of energy distributions deduced at several relative levels in figure 5a.]

sion ; ils sont ensuite analysés en masse et en énergie dans un spectromètre à double déviation, magnétique et électrostatique.

3.1 Collecte DES IONS SECONDAires. - Les caractéristiques de l'optique collectrice sont bien définies [8]. Rappelons que dès leur départ les ions sont accélérés par un fort champ électrostatique $E=V / D$ et que l'objectif complet forme simultanément une image agrandie de la surface sur le diaphragme d'ouverture $D_{0}$ du spectromètre et un «crossover» (pupille du système optique) sur son diaphragme d'entrée $d_{0}$ (voir Fig. 7); vis-à-vis de l'optique collectrice, $d_{0}$ et $D_{0}$ remplissent respectivement les rôles de diaphragme d'ouverture et de diaphragme de champ. On établit facilement que les trajectoires des ions émis avec une charge électrique élémentaire, une énergie initiale $\phi_{0}$ exprimée en

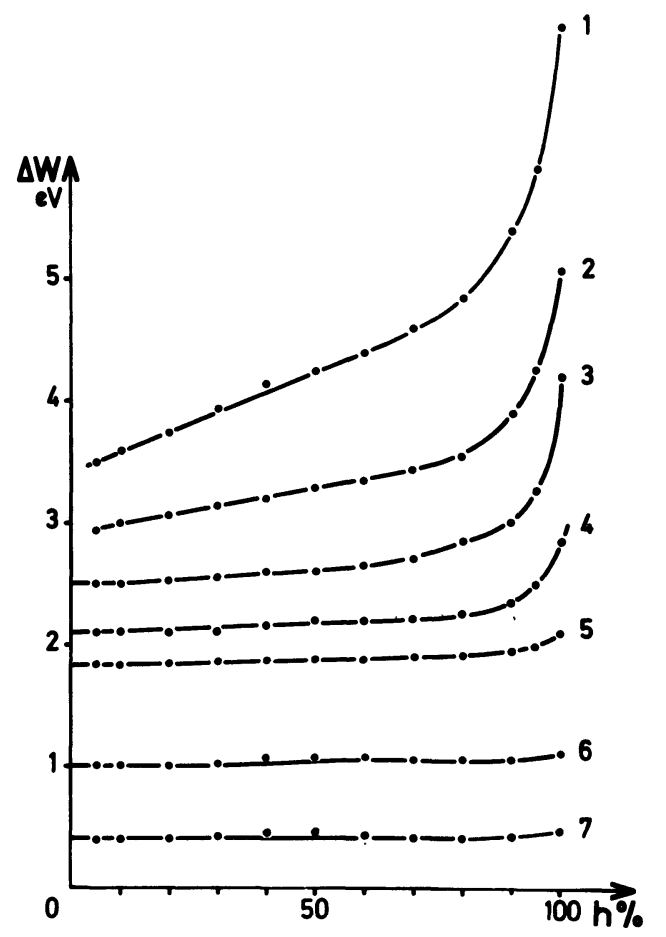

b)

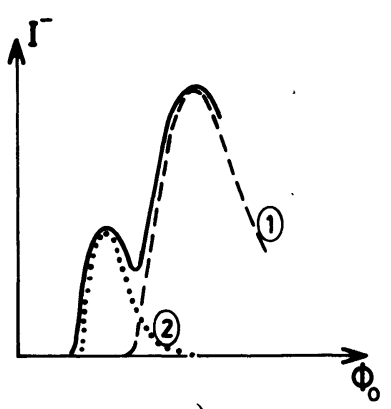

a)

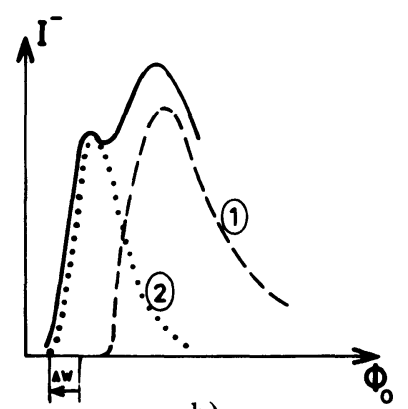

b)
Fig. 6. - Distributions énergétiques fictives où deux processus coexisteraient ; la détermination des variations de travail de sortie (Fig. 6b) serait alors fortement perturbée.

[Possible energy distributions which render impossible the determination of the work function variation (Fig. 6b).]

électron-volts, suivant une direction formant l'angle $\alpha_{0}$ avec l'axe optique recoupent le plan transversal du diaphragme d'entrée à la distance $\mathrm{CP}=r$ :

$$
r=4 D \sqrt{\phi_{0} / V} \sin \alpha_{0}
$$

où $V$ représente la tension appliquée à l'échantillon situé à la distance $D$ de l'électrode au potentiel de la masse. La comparaison de la taille du crossover avec le diamètre $d_{0}$ du diaphragme de contraste fixe directement les discriminations introduites par l'optique collectrice :

collecte totale $0<\alpha_{0}<90^{\circ}$ lorsque

$$
\phi_{0} \leq \phi_{0 \mathrm{M}}=\left(d_{0} / 8 D\right)^{2} V
$$




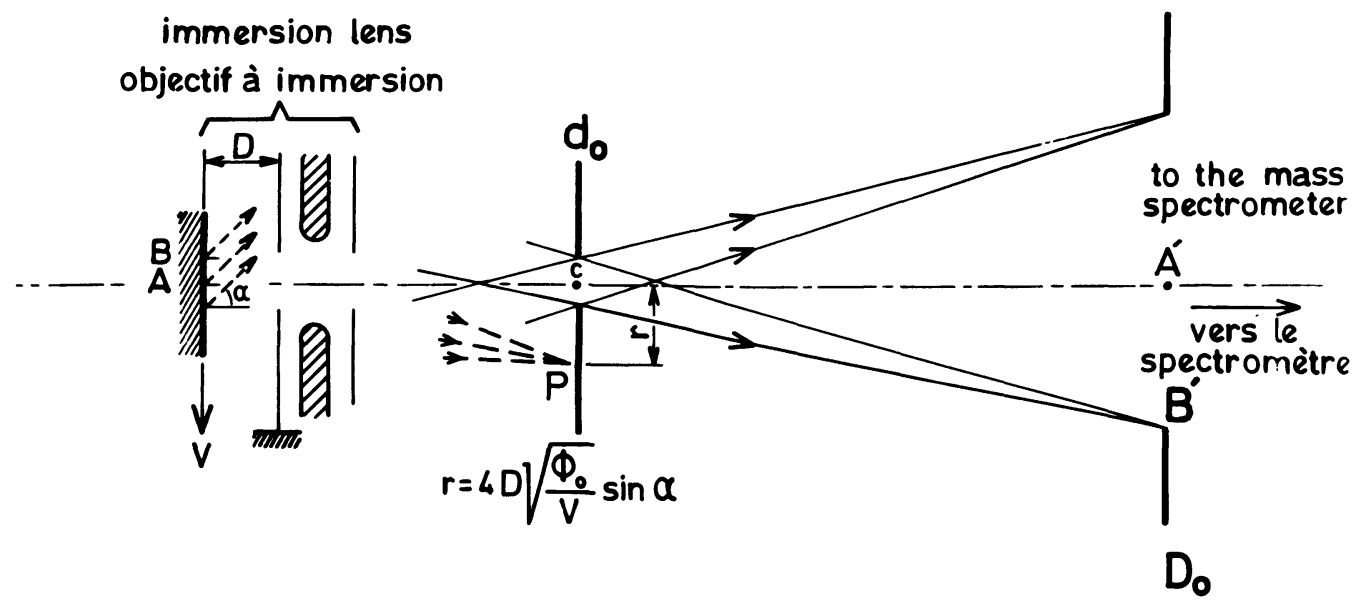

Fig. 7. - Schéma de l'optique à immersion qui collecte les ions secondaires.

[Optical system used for collecting the secondary ions.]

collecte partielle $\alpha_{0} \leq \alpha_{0 \mathrm{M}}$ si $\phi_{0}>\phi_{0 \mathrm{M}}$

$$
\text { avec } \sin \alpha_{0 M}=\left(\phi_{0 M} / \phi_{0}\right)^{1 / 2}
$$

$\phi_{0 \mathrm{M}}$ vaut $0,1 \mathrm{eV}$ lorsque $V=4000 \mathrm{~V}, d_{0}=0,1 \mathrm{~mm}$ et $D=5 \mathrm{~mm}$.

3.2 ANALYSE DES IONS SECONDAIRES. - Le système dispersif est constitué d'une association particulière de deux prismes à $90^{\circ}$ à double focalisation. Dans ce montage, l'analyse en quantité de mouvement dans le prisme magnétique est suivie d'une analyse en énergie dans le prisme électrostatique. Cette association, schématisée figure 8 , accroît la

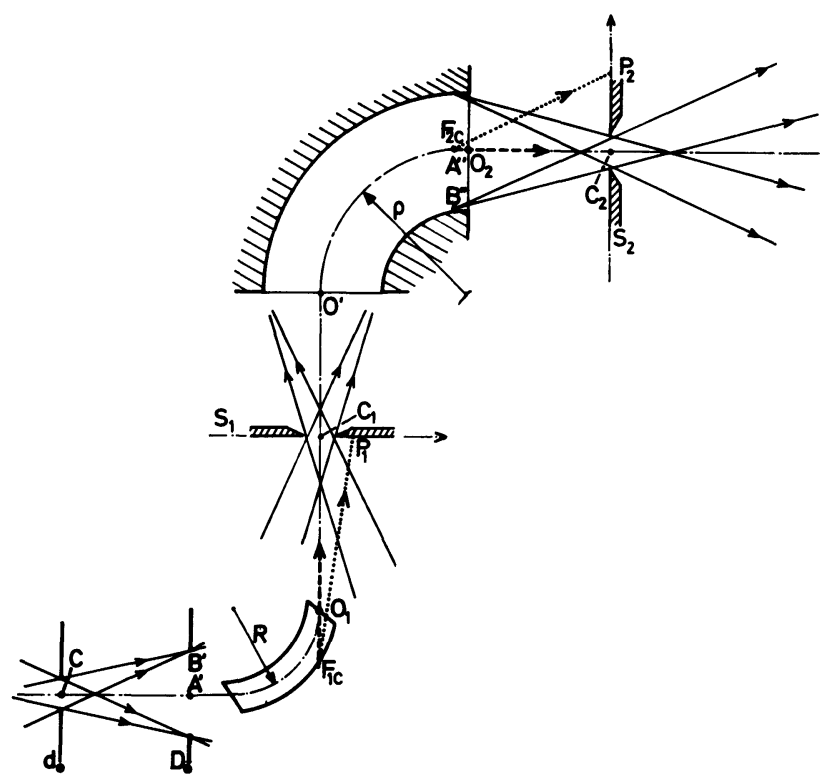

Fig. 8. - Schéma du système dispersif. La déviation magnétique correspond à un rayon $R=125 \mathrm{~mm}$; le prisme électrostatique de rayon $\rho=200 \mathrm{~mm}$ est placé à la distance $\mathrm{O}_{1} \mathrm{O}^{\prime}=\mathrm{O}_{1} \mathrm{C}_{1}+\mathrm{C}_{1} \mathrm{O}^{\prime}=2 R+\rho$ de la sortie du prisme magnétique, les lames de sélection $S_{2}$ de sortie sont situées en $\mathrm{C}_{2}$ sur l'image du diaphragme $d_{0}\left(\mathrm{O}_{2} \mathrm{C}_{2}=\rho\right)$.

[Schema of the used mass spectrometer.] dispersion en énergie ; elle convient pour l'examen précis des distributions énergétiques et est donc bien adaptée à l'étude des processus d'ionisation. (Une autre disposition permet d'augmenter la résolution en masse ; elle est souvent préférée dans les dispositifs d'analyse).

Les images du diaphragme d'entrée $d_{0}$ sont projetées au grandissement unité sur les fentes de sélection $S_{1}$ et $S_{2}$. Le calcul montre qu'une légère modification $\Delta V$ de la tension d'accélération $V$ produit un pivotement des trajectoires centrales autour des foyers chromatiques $F_{1 \mathrm{c}}$ (de la déviation magnétique seule) et $\mathrm{F}_{2 \mathrm{c}}$ (de l'ensemble du spectromètre).

$$
\mathrm{F}_{1 \mathrm{c}} \mathrm{O}_{1}=2 R / 3 \text { et } \mathrm{F}_{2 \mathrm{c}} \mathrm{O}_{2}=\rho /(7+8 R / \rho)
$$

(les rayons $R$ et $\rho$ sont définis sur la figure 8).

Dans le plan des lames de sélection $S_{1}$ et $S_{2}$, les dispersions en énergie valent respectivement :

et

$$
\begin{aligned}
& \mathrm{C}_{1} \mathrm{P}_{1}=4 \Delta R=2 R \Delta V / V \\
& \mathrm{C}_{2} \mathrm{P}_{2}=2(R+\rho) \Delta V / V .
\end{aligned}
$$

Il en résulte que si le faisceau était réduit à sa trajectoire centrale, des fentes de sélection successives $S_{1}$ et $S_{2}$ de largeur $\ell$ isoleraient des bandes passantes :

et

$$
\begin{aligned}
& \Delta V_{1}=V \ell / 2 R \\
& \Delta V_{2}=V \ell / 2(R+\rho) .
\end{aligned}
$$

Mais pour conserver une intensité aux faisceaux transmis, on doit associer un diaphragme de contraste de diamètre $d_{0}$ à une ouverture $\ell$ des lames de sélection égale ou légèrement supérieure à $d_{0}$ (de façon à prendre en compte les aberrations d'ouverture du spectromètre). La séparation de deux énergies voisines ne devient totale que lorsque les écarts $\Delta V_{1}^{\prime}$ et $\Delta V_{2}^{\prime}$ s'élèvent au double des bandes passantes précédentes : 


$$
\text { et } \quad \begin{aligned}
& \Delta V_{1}^{\prime}=V \ell / R \\
& \Delta V_{2}^{\prime}=V \ell /(R+\rho) .
\end{aligned}
$$

La relation (3) fixe les positions des images de la pupille d'entrée projetées dans le plan de la fente de sélection $\mathrm{S}_{2}$ : leurs positions relatives varient avec l'énergie initiale $\phi_{0}=\Delta V$ et la tension d'accélération $V$; leurs positions absolues dépendent des tensions de polarisation du prisme électrostatique et de l'induction magnétique (voir paragraphe 5).

Quelques valeurs sont reportées tableau I.

3.3 ObSERVATION DES images Filtrées ET MESURE. - L'ensemble du spectromètre transpose l'image initiale $A^{\prime} B^{\prime}$ de la surface en $A^{\prime \prime} B^{\prime \prime}$ au niveau de la focale chromatique $F_{2 c}$. Un système de deux lentilles électrostatiques projette une image définitive agrandie de la surface sur la face d'entrée d'un amplificateur de brillance à galette de micro-canaux. Pour les mesures d'intensité, le faisceau d'ions secondaires peut être également focalisé sur l'entrée d'un multiplicateur à électrons secondaires; une post-accélération des ions avant leur conversion permet des comptages avec de très bons rendements quantiques, même pour les ions négatifs.

3.4 RELEVÉ DES DISTRIBUTIONS EN ÉNERGIE. - La méthode retenue ici consiste à faire défiler les spectres d'énergie devant les fentes de sélection en modifiant le potentiel d'accélération des ions de telle sorte que l'énergie des ions qui traversent le spectromètre reste constante ; dans ces conditions, il n'est pas nécessaire de modifier les réglages des prismes du spectromètre puisque l'énergie initiale $\phi_{0}$ des ions isolés compense juste le défaut d'accélération.

Décomposons la tension appliquée en une tension fixe $V$ et une tension variable $-v$; l'énergie cinétique $U$ des ions négatifs à la sortie de l'optique accélératrice en électrons-volts s'exprime par :

$$
U=V-v+w+\phi_{0}
$$

où $w$ représente la différence de potentiel de contact entre la surface de l'échantillon et l'enceinte du spectromètre et $\phi_{0}$ l'énergie initiale des ions exprimée en $\mathrm{eV}$. Dans le cas d'ions de charge égale à $n$ fois la charge élémentaire $\phi_{0}$ devrait être remplacé par $\phi_{0} / n$.

La figure 9 résume comment la variation de la tension $-v$ conduit à l'enregistrement direct des distributions énergétiques. L'énergie des ions isolés restant fixe, la bande passante énergétique demeure invariante ; une alimentation électrique distincte de l'alimentation de polarisation de l'échantillon permet d'éviter des défocalisations du faisceau secondaire pendant ces relevés. La gamme des énergies initiales explorées est limitée à une centaine d'électrons-volts environ; les variations de tension additionnelle doivent en effet, rester petites devant la tension totale d'accélération pour éviter d'introduire des déviations gênantes sur le faisceau d'ions primaires.

Dans ces relevés de distributions énergétiques, il faut assurer au préalable un centrage commun des

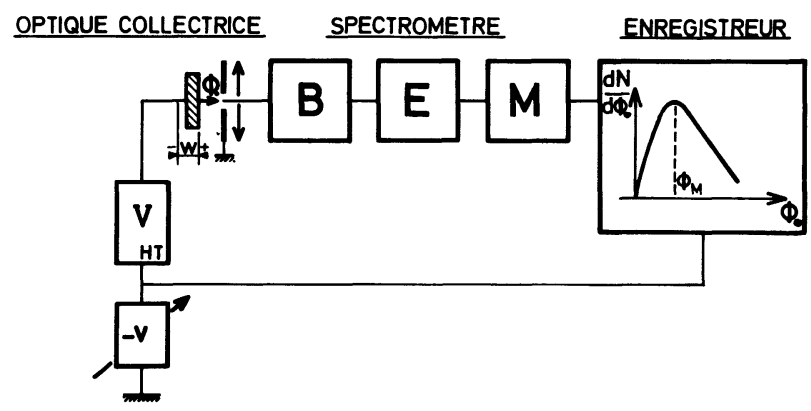

Fig. 9. - Schéma de principe du relevé direct des distributions énergétiques. Le spectromètre représenté symboliquement par la succession du prisme magnétique (B), du prisme électrostatique (E) et de l'élément de mesure $(\mathrm{M})$ conserve un réglage fixe ; il isole des ions d'énergie $U=V-v+w+\phi_{0}$; le balayage de la tension additionnelle $-v$ permet d'isoler les ions dont l'énergie initiale $\phi_{0}$ compense exactement les variations de tension accélératrice.

[Direct recording of the ion energy distributions. B and $\mathrm{E}$ represent the magnetic and electrostatic prisms respectively.]

Tableau I.

$$
d_{0}=200 \mu \mathrm{m} \quad \ell=250 \mu \mathrm{m}
$$

\begin{tabular}{|l|c|c|c|c|}
\cline { 2 - 5 } \multicolumn{1}{c|}{} & $\begin{array}{c}\text { Tension } \\
\text { d'accélération } \\
\mathrm{V}\end{array}$ & $\begin{array}{c}\text { Dispersion } \\
\mu \mathrm{m} / \mathrm{V}\end{array}$ & $\begin{array}{c}\text { Bande } \\
\text { passante } \\
\mathrm{eV}\end{array}$ & $\begin{array}{c}\text { Ecart des } \\
\text { énergies } \\
\text { résolues } \mathrm{eV}\end{array}$ \\
\hline $\begin{array}{c}\text { Prisme magnétique } \\
\text { seul }\end{array}$ & 1000 & 250 & 1 & 2 \\
\hline Ensemble du & 4000 & 645 & 4 & 8 \\
\hline spectromètre & 1000 & 650 & 0,38 & 0,76 \\
\hline
\end{tabular}


bandes passantes des deux prismes. On l'effectue habituellement au niveau de l'énergie initiale la plus probable $\phi_{\mathrm{M}}$. Mais, comme cette énergie n'est pas connue a priori on doit utiliser des ajustements successifs des alimentations de tension additionnelle $v$ et de champ magnétique pour rendre maximum l'intensité du faisceau transmis dans le système spectrométrique complet.

Mais ce réglage, s'il facilite grandement l'enregistrement des distributions énergétiques, ne permet ni un repérage absolu du début des émissions ni une comparaison précise des distributions d'espèces ioniques différentes. L'énergie $\phi_{M}$ d'émission la plus probable peut en effet changer d'une espèce à l'autre. Cette situation est observée pour les ions $\mathrm{Cu}^{-}$et $\mathrm{O}^{-}$émis par un monocristal d'oxyde cuivreux, suffisamment conducteur pour que sa surface reste parfaitement équipotentielle; dans ce cas une comparaison utilisant les réglages précédents pourrait laisser supposer que les distributions énergétiques correspondantes ne débutent pas à partir d'une même et très faible énergie initiale et la fixation de césium pourrait alors produire des effets analogues à ceux imaginés sur la figure 6 , il n'en est rien (voir paragraphe 5).

\section{Relevé des variations de travail de sortie.}

Comme nous l'avons indiqué plus haut, un changement de travail de sortie au cours de réactions superficielles produit une variation de potentiel de contact répercutée par un décalage des distributions énergétiques des ions secondaires.

Cette possibilité de mesure tient au fait que les réactions superficielles, limitées à une faible portion de la surface de l'échantillon, n'affectent pas les surfaces de référence (carter du prisme magnétique et électrodes du prisme électrostatique protégées par leur éloignement de l'échantillon et par un pompage différentiel). Le jet de vapeur de césium couvre une plage de $600 \mu \mathrm{m}$ de diamètre bien plus petite que la surface bombardée par les ions primaires $(3 \mathrm{~mm}$ de diamètre), mais qui déborde largement de part et d'autre du champ imagé par l'objectif $(200 \mu \mathrm{m})$.

Naturellement, la localisation du dépôt de césium crée un changement de travail de sortie sur une portion seulement de la surface de l'échantillon [3]. En bordure de cette zone, il apparaît des champs électriques transversaux qui peuvent affecter les trajectoires initiales des ions secondaires émis au voisinage de cette limite. Mais dans notre montage, ce champ électrique local vient se superposer à un champ uniforme quelque mille fois plus intense; l'effet résultant est d'autant moins perceptible qu'un centrage correct du jet de césium conduit à examiner des plages éloignées de la bordure. De plus, si l'influence du champ électrique local n'avait pas été négligeable dans notre montage, il aurait pu intro- duire aussi des défocalisations sur les micrographies ioniques d'échantillons polycristallins (mais on n'a jamais observé ces défocalisations bien que les différences de travail de sortie de grain à grain atteignent souvent $0,5 \mathrm{eV}$ ). Mais il est vraisemblable que les mesures puissent être davantage perturbées dans les dispositifs ne comportant qu'un très faible champ extracteur, que le faisceau utilisé pour la détermination des variations de travail de sortie soit un faisceau d'ions secondaires ou un faisceau auxiliaire d'électrons.

Examinons la détermination pratique des variations de travail de sortie par le relevé du décalage des distributions énergétiques pour les ions de très basse énergie initiale. Montrons comment une précision meilleure que $0,1 \mathrm{eV}$ est facilement atteinte bien que la bande passante du spectromètre soit bien plus élevée (voir Tab. I).

Lorsque l'énergie initiale augmente, la taille des crossovers projetés sur les fentes s'accroît progressivement jusqu'à ce qu'intervienne la limitation par le diamètre du diaphragme de contraste $d_{0}$ (au-delà de l'énergie $\left.\phi_{0 \mathrm{M}}\right)$. Les propriétés dispersives du spectromètre sont telles que les différents crossovers présentent dans le plan des lames de sélection un décalage géométrique fixé par la dispersion en énergie du montage. Les figures $10 \mathrm{a}$ et $10 \mathrm{~b}$ schématisent les positions respectives pour deux accélérations des ions à 1000 et $4000 \mathrm{eV}$. Une modification de la tension additionnelle $v$, une légère modification du champ magnétique ou une variation de travail de sortie (induite par un changement de composition superficielle) déplace globalement l'ensemble des crossovers dans le plan des lames de sélection mais conserve leurs positions respectives. Dans la zone de croissance initiale rapide, qui correspond aux ions de faibles énergies initiales $\left(\phi_{0}<\phi_{M}\right)$, un petit décalage se traduit par une forte variation de l'intensité transmise. La détermination gagne donc en précision. En effet, les variations d'intensité observées dépendent alors directement de la dispersion énergétique du montage mais pas de sa bande passante, puisque seule intervient la coupure par la lame qui élimine les ions de haute énergie. Ainsi la mesure des décalages des distributions énergétiques est limitée surtout par la stabilité géométrique du faisceau au niveau des fentes de sélection et donc par les instabilités des alimentations qui polarisent l'échantillon, les électrodes du prisme électrostatique et par la régulation du champ magnétique. Des stabilités élémentaires meilleures que $1 \times 10^{-5}$ permettent une mesure des variations de travail de sortie à mieux que $0,1 \mathrm{eV}$ près. Cette précision est donc tout à fait comparable à celle obtenue avec un spectromètre à miroir électrostatique assurant un filtrage par contre-champ beaucoup plus fin pour les basses énergies initiales [2].

On peut remarquer sur les figures $10 \mathrm{a}$ et $10 \mathrm{~b}$ que 


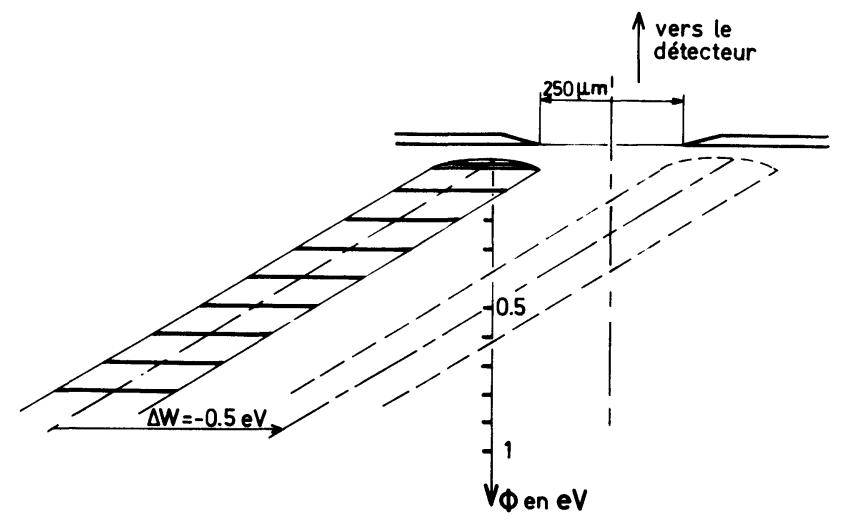

a)

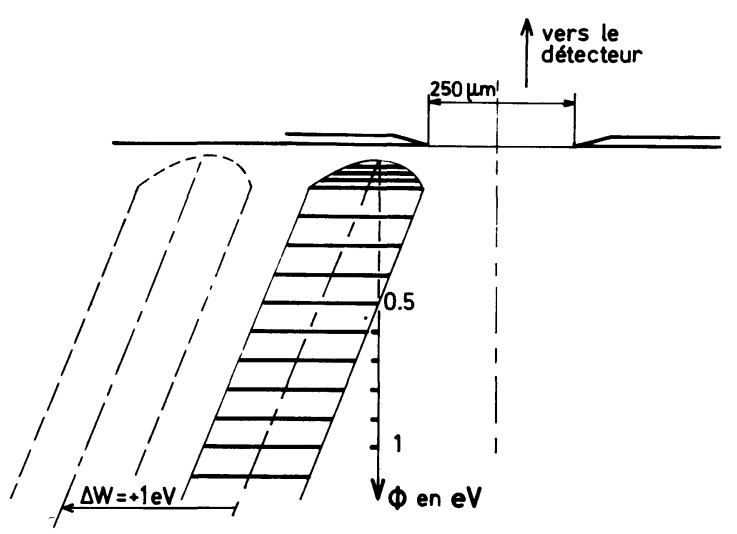

b)

Fig. 10. - a) Représentation des positions respectives des crossovers au niveau des lames de sortie. L'accélération des ions à $1000 \mathrm{eV}$ conduit à une dispersion de $650 \mu \mathrm{m} / \mathrm{V}$, le diaphragme $d_{0}=200 \mu \mathrm{m}$ et l'ouverture de la fente $\mathrm{S}_{2}=250 \mu \mathrm{m}$ correspondent à une bande passante égale à $0,4 \mathrm{eV}$. L'enveloppe des crossovers représentée en trait interrompu reproduit une diminution de $0,5 \mathrm{eV}$ du travail de sortie de l'échantillon; les crossovers correspondant aux ions de 0,3 et $0,4 \mathrm{eV}$ sont alors totalement transmis alors qu'en position initiale aucun faisceau ne franchissait les lames. b) Représentation des positions respectives des crossovers projetés au niveau des lames de sortie. Une accélération des ions sous $4000 \mathrm{~V}$ conduit à une dispersion de $160 \mu \mathrm{m} / \mathrm{V}$, un diaphragme de contraste $d_{0}$ de $200 \mu \mathrm{m}$ de diamètre associé à une fente de sortie de $250 \mu \mathrm{m}$ fournit une bande passante de $1,5 \mathrm{eV}$ et une résolution de $3 \mathrm{eV}$. L'enveloppe des crossovers représentée en trait interrompu correspond à une élévation de $1 \mathrm{eV}$ du travail de sortie. Les aberrations d'ouverture du spectromètre, qui peuvent élargir légèrement les crossovers, n'ont pas été prises en compte sur ces tracés.

[a) Crossover positions for increasing initial energies for $U=1000 \mathrm{~V}$. The displaced envelope corresponds to a $0.5 \mathrm{eV}$ shift of the sample work function. b) Crossover positions for $U=4000 \mathrm{~V}$. The dispersion reaches $160 \mu \mathrm{m} / \mathrm{V}$, the energy band pass $1.5 \mathrm{eV}$ and the energy resolution is $3 \mathrm{eV}$.]

le début des faisceaux transmis est constitué par des ions d'énergie initiale très faible mais non nulle $(25 \mathrm{meV}$ et $100 \mathrm{meV})$. L'origine des émissions est donc repérée avec une très légère erreur systématique égale à l'énergie $\phi_{0 \mathrm{M}}$ limite de la collecte totale ; toutefois, proportionnelle à la tension d'accélération et au carré du diamètre du diaphragme de contraste, cette erreur reste indépendante des réactions chimiques à la surface des échantilons; la raideur des croissances des distributions s'en trouve accentuée, ce qui facilite donc la détermination des variations de travail de sortie.

Il n'est pas exclu que l'adsorption d'atomes lourds, comme le césium, sur des substrats plus légers puisse changer les distributions angulaires des atomes éjectés par la pulvérisation. Mais ces modifications n'affectent sans doute que peu les distributions des ions de très basse énergie totalement collectés par l'optique à immersion.

\section{Comparaison précise de distributions énergétiques d'espèces ioniques différentes.}

Nous avons décrit au paragraphe 3.4 comment le balayage de la tension additionnelle permettait un relevé rapide des distributions en énergie. Lors de ces enregistrements, l'origine des énergies initiales se trouvait ainsi fixée avec une précision de l'ordre de $1 \mathrm{eV}$, voisine de la bande passante du spectromètre. Mais cette précision devient nettement insuffisante pour assurer une comparaison plus fine des distributions énergétiques d'espèces ioniques différentes ou pour examiner si l'émission secondaire présente une origine énergétique commune pour chaque espèce ionique.

Rappelons que dans notre montage, les deux prismes contribuent à la dispersion en énergie. Il en résulte que l'origine des énergies initiales dépend de façon apparente du réglage du prisme magnétique. La principale difficulté, pour atteindre la précision recherchée, réside précisément dans ce dernier réglage qui fixe la masse et l'énergie des ions dirigés suivant l'axe d'entrée du prisme électrostatique.

\subsection{AJUSTAGE DU PRISME MAGNÉTIQUE AVEC UN} FAISCEAU D'IONS. - Pour isoler l'influence de chaque déviation on peut déterminer par exemple avec grande précision les deux champs magnétiques $B_{1}$ et $B_{2}$ qui assurent des déviations identiques des ions ${ }^{16} \mathrm{O}^{-}$et ${ }^{63} \mathrm{Cu}^{-}$de même énergie en associant mesure du champ magnétique et calcul (rappelons que $B=143,6 \sqrt{M U / n}$. $R$ où le champ magnétique $B$ est exprimé en gauss, le rayon $R$ en $\mathrm{m}, M$ et $n$ représentent la masse et la charge de l'ion en unités atomiques et $U$ son énergie en électrons-volts). Mais les défauts de linéarité de la sonde Hall ne permettent pas d'atteindre la précision recherchée.

Or, vis-à-vis du champ magnétique une variation de la masse $M$ ou une variation de l'énergie $U$ jouent un rôle équivalent pourvu que le produit $M U$ soit conservé. Les deux champs magnétiques recherchés 
$B_{1}$ et $B_{2}$ sont donc identiques à ceux qui assurent les déviations des ions ${ }^{16} \mathrm{O}^{-}$accélérés sous les tensions respectives $1000,00 \mathrm{~V}$ et $3934,06 \mathrm{~V}$ (le rapport de ces tensions est déduit des tables de masses atomiques [9] en incluant la masse des électrons apportés lors de l'ionisation) : pour déterminer expérimentalement ces champs magnétiques, on isole la déviation magnétique, en relevant les intensités sur un cylindre de Faraday intermédiaire :

Pour conserver, dans cette variation d'énergie, à la fois la bande passante et l'efficacité de collecte, il faut ajuster la largeur $\ell$ de la fente de sélection en raison inverse de la tension appliquée (d'après (2)) et accroître le diamètre du diaphragme de contraste comme l'inverse de sa racine carrée (d'après (1)). Le réglage du champ magnétique pour le second enregistrement est donc effectué de façon à assurer une bonne superposition des distributions (voir Fig. 11).

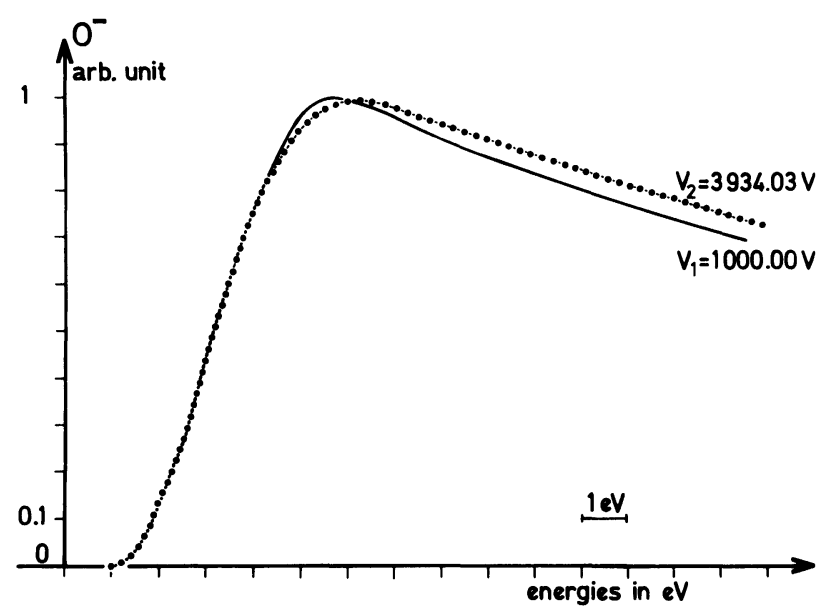

Fig. 11. - Ajustement du prisme magnétique pour assurer des déviations identiques des ions ${ }^{63} \mathrm{Cu}^{-}$et ${ }^{16} \mathrm{O}^{-}$accélérés sous $1000 \mathrm{eV}$. Pour ce réglage, on utilise des ions ${ }^{16} \mathrm{O}^{-}$accélérés sous les tensions respectives $3934,06 \mathrm{~V}$ et $1000,00 \mathrm{~V}$.

[Preliminary magnetic prism ajustment for identical ${ }^{63} \mathrm{Cu}^{-}$and ${ }^{16} \mathrm{O}^{-}$deviation. Here the ${ }^{16} \mathrm{O}^{-}$ions used were accelerated to $3934.06 \mathrm{~V}$ and $1000.00 \mathrm{~V}$.]

Par la suite, la reproduction de ces réglages de champ magnétique est facilitée par une mesure précise des tensions Hall par comparaison avec un standard de tension. Les enregistrements définitifs reportés figure 12 sont effectués à l'aide du spectromètre complet pour une tension d'accélération fixe en choisissant cette fois un diaphragme de contraste et une ouverture des fentes de sélection qui assurent une haute résolution en énergie. Ainsi ce double enregistrement établit que l'émission débute à partir d'une énergie initiale très faible identique pour chacune des deux espèces ${ }^{16} \mathrm{O}^{-}$et ${ }^{63} \mathrm{Cu}^{-}$(9). On peut remarquer sur la figure 12 les différences de

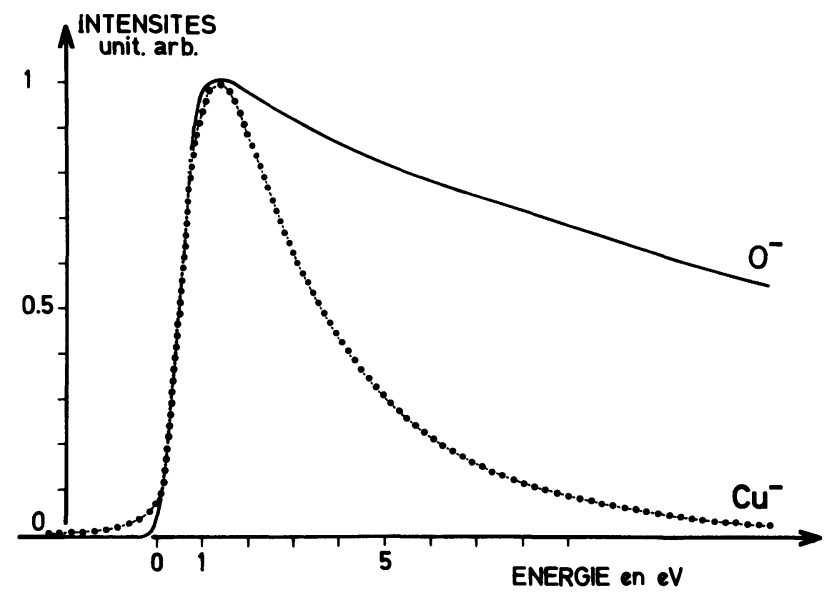

Fig. 12. - Comparaison des distributions des ions ${ }^{63} \mathrm{Cu}^{-}$et ${ }^{16} \mathrm{O}^{-}$émis par un cristal d'oxyde cuivreux $\left(d_{0}=200 \mu \mathrm{m} ; \ell=250 \mu \mathrm{m} ; V=1000 \mathrm{~V}\right)$. La bande passante $\Delta V_{2}$ vaut $0,4 \mathrm{eV}$ et $\phi_{0 \mathrm{M}} 0,03 \mathrm{eV}$.

[Comparison of ${ }^{63} \mathrm{Cu}^{-}$and ${ }^{16} \mathrm{O}^{-}$energy distributions measured on the copper crystal.]

forme des distributions énergétiques qu'on accentue nettement lorsque l'on utilise des diaphragmes de contraste plus grands. Notons aussi une légère production d'ions $\mathrm{Cu}^{-}$par des processus d'ionisation retardés. Des réglages analogues conduisent à une comparaison similaire des émissions ${ }^{18} \mathrm{O}^{-}$et ${ }^{65} \mathrm{Cu}^{-}$. Dans ces opérations, le repérage préalable des champs magnétiques fixe la précision de la méthode. Cette précision dépend naturellement de la qualité des mouvements de diaphragmes et d'ouverture de la fente de sélection, ainsi que de la taille des diaphragmes disponibles. Une mesure directe très précise des champs magnétiques par sonde RMN permettrait un ajustage instrumental direct.

\subsection{COMPARAISON À CHAMP MAGNÉTIQUe FIXE.-} La seconde méthode expérimentée utilise cette fois le montage spectrométrique complet avec un champ magnétique fixe. La modification de la tension accélératrice est accompagnée cette fois d'un ajustement des polarisations du prisme électrostatique.

Sur l'échantillon d'oxyde de cuivre précédent, nous avons obtenu une coïncidence à $0,1 \mathrm{eV}$ près pour le début des distributions des ions ${ }^{16} \mathrm{O}^{-}$, ${ }^{18} \mathrm{O}^{-}$et ${ }^{19} \mathrm{~F}^{-}$, d'une part, ainsi que $\mathrm{CuO}^{-}$(masses 79 et 81) ; mais la précision décroît pour les comparaisons des ions ${ }^{16} \mathrm{O}^{-}$et ${ }^{35} \mathrm{Cl}^{-}$ainsi que ${ }^{35} \mathrm{Cl}^{-}$et ${ }^{63} \mathrm{Cu}$ (coïncidence à $0,3 \mathrm{eV}$ ) et devient médiocre dans le cas de la comparaison directe des ions $\mathrm{O}^{-}$aux ions $\mathrm{Cu}^{-}$. Ces difficultés expérimentales sont la conséquence des imperfections de l'alignement instrumental; elles jouent d'autant plus que le rapport des masses des espèces ioniques est éloigné de l'unité. En appendice nous précisons la méthode utilisée pour parfaire le réglage à partir d'une légère 
correction de calibrage du prisme électrostatique. Ce réglage repose sur la mise en coïncidence du début des distributions des ions ${ }^{16} \mathrm{O}^{-}$et ${ }^{63} \mathrm{Cu}^{-}$, établie par les expériences reportées sur la figure 12 . Cet ajustement effectué, il devient facile de vérifier par de nouvelles modifications de tension d'accélération que la superposition du début des émissions s'étend à tous les ions émis par l'échantillon, notamment aux ions $\mathrm{CuO}^{-}, \mathrm{Cu}_{2} \mathrm{O}^{-}$et $\mathrm{Cl}^{-}$avec cette fois une précision excellente (égale à $0,1 \mathrm{eV}$ ).

Ainsi, les expériences établissent que l'ensemble des distributions des ions émis par l'échantillon d'oxyde cuivreux débutent à partir d'une même énergie initiale très faible et vraisemblablement nulle. Mais il n'existe pas d'éléments qui permettent d'affirmer que, d'une façon générale, l'émission d'ions secondaires commence dès les énergies initiales nulles. Seul un calibrage très précis du spectromètre, prenant parfaitement en compte toutes les différences de potentiel de contact, permettrait de le démontrer. Il reste cependant difficile d'imaginer des raisons physiques qui introduiraient un effet de seuil.

Pour appliquer le second procédé à la comparaison de distributions énergétiques, il est indispensable d'accompagner la variation de tension accélératrice d'une modification du diaphragme de contraste et de la largeur de fente de sélection, tandis que pour le premier procédé proposé, ces modifications n'intervenaient que dans le réglage instrumental préalable. Mais cette commutation sous champ magnétique fixe permet facilement, après un réglage unique, de comparer les travaux de sortie de plusieurs échantillons sans nécessiter l'enregistrement de distribution de la même espèce ionique. La cohérence des mesures entre elles reste très bonne toutes les fois que plusieurs espèces sont utilisées pour le même échantillon. A titre d'exemple, les mesures reportées figure 13 sont en bon accord avec les données de la littérature $[2,10]$ sauf pour l'arséniure de gallium où des changements de stœchiométrie de surface dus à

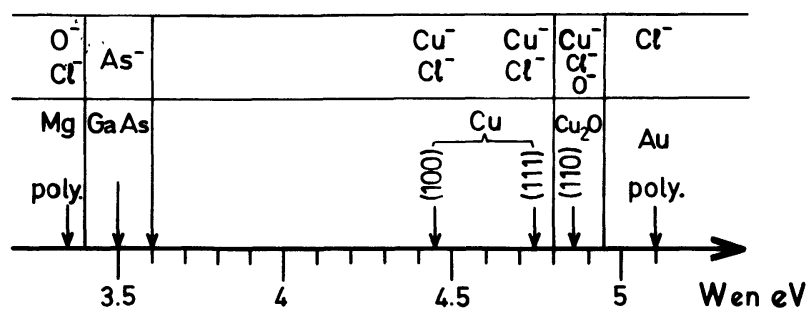

Fig. 13. - Mesures des travaux de sortie sur différents échantillons à partir des distributions de plusieurs espèces ioniques. La valeur absolue pour l'or, $5,1 \mathrm{eV}$ a été extraite des tables [11] pour servir de référence.

[Comparison of some work functions measured on different samples with several ion species. The reference value chosen for the gold sample, $5.1 \mathrm{eV}$, was taken from table [11].]

REVUE DE PHYSIQUe APPLIQUÉE. - T. 24, N 5, MAI 1989 l'érosion ionique réduisent sans doute le travail de sortie.

\section{Conclusion.}

Les expériences que nous avons présentées permettent de préciser les bases de la détermination des variations de travail de sortie à partir du décalage des distributions énergétiques des ions secondaires. Ce procédé est conduit dans les conditions mêmes de l'analyse sans apporter aucune perturbation aux équilibres dynamiques constitués à la surface des échantillons par la fixation d'éléments réactifs. Mais, si les rendements d'ionisation sont fortement accrus par ces réactions superficielles, la forme des distributions énergétiques demeure peu modifiée pour les ions de basse énergie ; la mesure des décalages des distributions est alors grandement facilitée. Cette situation très favorable est due à la fois à l'emploi d'une optique collectrice à immersion dont l'efficacité reste proche de l'unité pour les ions de faible énergie et à une émission des ions secondaires dès les très basses énergies initiales. L'emploi d'un spectromètre fortement dispersif en énergie conduit alors à des mesures avec une précision meilleure que $0,1 \mathrm{eV}$, fixée davantage par la dispersion du montage et la stabilité des alimentations électriques que par la bande passante directe de l'appareil. De telles déterminations ont permis de préciser expérimentalement comment le travail de sortie influençait les rendements d'émission des ions négatifs formés en présence de césium et d'améliorer la connaissance des processus d'ionisation correspondants [6].

Rappelons en outre que la méthode de réglage instrumental peut être directement appliquée à la comparaison de distributions énergétiques d'espèces ioniques différentes, avec une précision de l'ordre de $0,1 \mathrm{eV}$ bien supérieure à la bande passante du spectromètre. L'étude des processus d'ionisation s'en trouvera ainsi grandement facilitée.

\section{Appendice.}

\section{Ajustement instrumental pour une comparaison par commutation de tension.}

Désignons par $V$ la tension totale d'accélération des ions, par $U_{\mathrm{a}}=V+u_{\mathrm{a}}$ et $U_{\mathrm{e}}=V+u_{\mathrm{e}}$ l'énergie des ions qui décrivent les trajectoires centrales des prismes magnétiques et électrostatiques, en incluant dans $u_{\mathrm{a}}$ et $u_{\mathrm{e}}$ les énergies initiales des ions et les différences de potentiel de contact.

Les propriétés dispersives du prisme magnétique (voir Fig. 14) sont telles qu'une particule d'énergie repérée par la variable $\nu$ telle que $U=V+\nu$ aborde la face d'entrée du prisme électrostatique en $x_{0}$ avec une inclinaison $x_{0}^{\prime}$ :

$x_{0}=-\frac{3}{4} \frac{\nu-u_{\mathrm{a}}}{U}\left(\frac{8 R}{3}+\rho\right) x_{0}^{\prime}=-\frac{3}{4} \frac{\nu-u_{\mathrm{a}}}{U}$. 


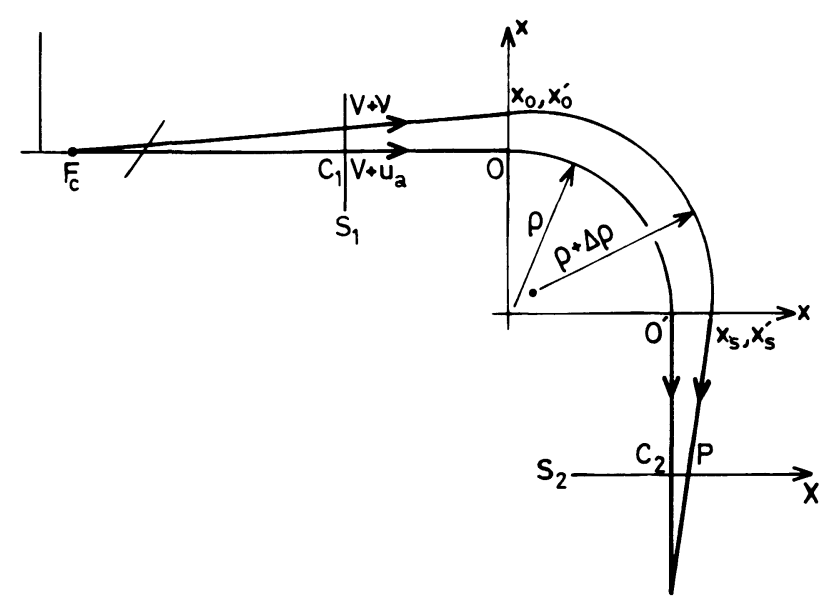

Fig. 14. - Examen de l'effet d'une imperfection d'alignement électrique du spectromètre.

[Influence of an electrical misalignment of the mass spectrometer.]

Ces relations résultent du pivotement de la trajectoire centrale des ions d'énergie $U=V+\nu$ autour de la focale chromatique $F_{1 \mathrm{c}}$, d'un angle $x_{0}^{\prime}=-\frac{3}{2} \frac{\Delta R}{R} \quad$ avec $\frac{\Delta R}{R}=\frac{1}{2} \frac{\Delta u}{U}, \Delta U=\nu-u_{\mathrm{a}}$ et $\mathrm{F}_{1 \mathrm{c}} \mathrm{O}=\frac{2}{3} R+2 R+\rho$.

Les relations de conjugaison optique dans le prisme électrostatique fournissent l'abscisse et l'inclinaison du point de sortie

$$
x_{\mathrm{s}}=x_{0}^{\prime} \rho+\Delta \rho \quad x_{\mathrm{s}}^{\prime}=-\frac{x_{0}}{\rho}+\frac{\Delta \rho}{\rho} \quad \text { avec } \quad \frac{\Delta \rho}{\rho}=\frac{\Delta U}{U}
$$

d'où l'intersection dans le plan de la fente de sortie :

$$
\mathrm{C}_{2} \mathrm{P}=x_{\mathrm{s}}+\rho x_{\mathrm{s}}^{\prime}=\rho x_{0}^{\prime}+2 \Delta \rho-x_{0}
$$

soit, d'après (8) et (9)

$$
X=\mathrm{C}_{2} \mathrm{P}=\frac{\nu-u_{\mathrm{a}}}{U} 2 R+\frac{\nu-u_{\mathrm{e}}}{U} 2 \rho .
$$

Il en résulte que l'énergie des ions isolée au centre de la fente est telle que :

$$
\nu=\frac{u_{\mathrm{e}}+u_{\mathrm{a}} R / \rho}{1+R / \rho}
$$

On isole ainsi les ions repérés par la tension $\nu$ et non ceux repérés par $u_{\mathrm{e}}$, le défaut d'alignement électrique $\mathrm{du}$ spectromètre introduit une erreur:

$$
u_{\mathrm{e}}-\nu=\left(u_{\mathrm{e}}-u_{\mathrm{a}}\right) \frac{R}{R+\rho} .
$$

Ajoutons un léger défaut d'alignement géométrique, défini par l'angle $\alpha$. Les ions d'énergie $V+\nu$ abordent le prisme électrostatique suivant :

$$
x_{0}=h+\rho \alpha \quad x_{0}^{\prime}=-\frac{3}{4} \frac{\nu-u_{\mathrm{a}}}{U}+\alpha
$$

avec $h=-\frac{3}{4}\left(\frac{8 R}{3}+\rho\right) \frac{\nu-u_{\mathrm{a}}}{U}$ dans la mesure où $\alpha$ et $\nu-u_{\mathrm{a}}$ restent petits

d'où $X=\frac{\nu-u_{\mathrm{a}}}{v} 2 R+\frac{\nu-u_{\mathrm{e}}}{v}$, relation indépendante de $\alpha$ identique à (9).

Ainsi, le pivotement du prisme électrostatique autour des lames de sélection $S_{1}$ ne change pas l'énergie isolée au centre des lames $S_{2}$ tant que l'inclinaison du faisceau de sortie reste compatible avec l'ouverture des faisceaux acceptés par l'optique de projection.

Examinons les effets d'une commutation de tension pour isoler tour à tour les ions de masse $M_{1}$ et $M_{2}$ accélérés respectivement par les tensions $V_{1}$ et $V_{2}$ telles que $M_{1} V_{1}=M_{2} V_{2}$, l'invariance du champ magnétique entraîne :

$M_{1}\left(V_{1}+u_{\mathrm{a}_{1}}\right)=M_{2}\left(V_{2}+u_{\mathrm{a}_{2}}\right)$ d'où

$M_{1} u_{\mathrm{a}_{1}}=M_{2} u_{\mathrm{a}_{2}}$.

La correction des alimentations du prisme électrostatique entraîne :

$V_{2}+u_{\mathrm{e}_{2}}=\frac{M_{1}}{M_{2}}\left(V_{1}+u_{\mathrm{e}_{1}}\right)$ d'où

$M_{1} u_{\mathrm{e}_{1}}=M_{2} u_{\mathrm{e}_{2}}$.

Les énergies isolées successivement au centre des lames sont $V_{1}+\nu_{1}$ et $V_{2}+\nu_{2}$ avec d'après (11)

$$
\nu_{1}=\frac{u_{\mathrm{c}_{1}}+u_{\mathrm{a}_{1}} R / p}{1+R / p} \text { et } \nu_{2}=\frac{u_{\mathrm{e}_{2}}+u_{\mathrm{a}_{2}} R / p}{1+R / p} .
$$

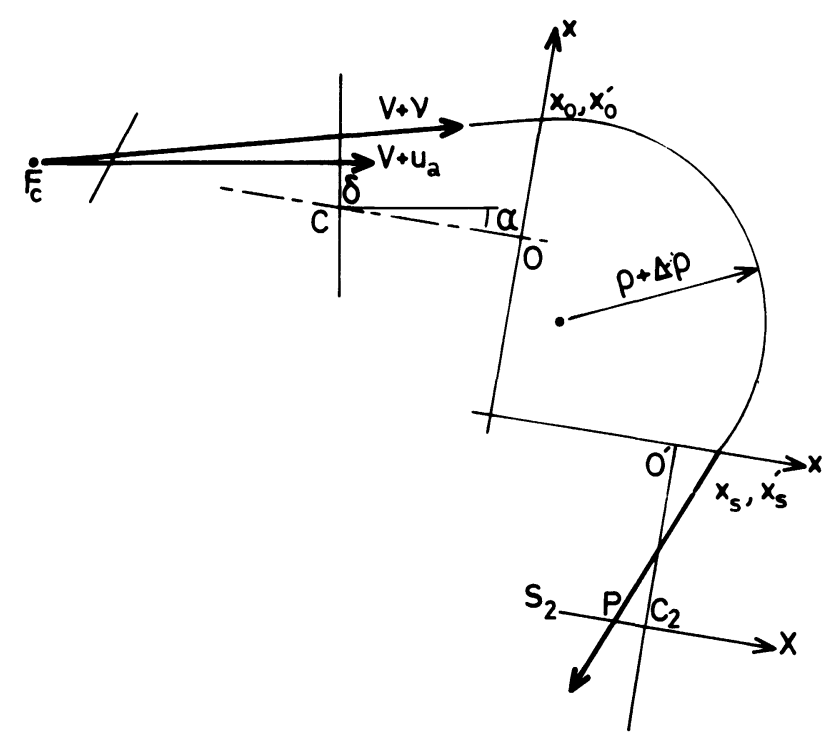

Fig. 15. - Examen d'un défaut d'alignement géométrique additionnel.

[Influence of additional geometrical misalignment.] 
Compte tenu des expressions (12) et (13) :

$$
\nu_{2}-\nu_{1}=\left(M_{1} / M_{2}-1\right) \nu_{1} \text {. }
$$

Cette dernière expression montre que la coïncidence des débuts de deux distributions $\nu_{2}-\nu_{1}=0$ n'est donc obtenue que lorsque $\nu_{1}=0$, ce qui nécessite d'après (11)

$$
u_{\mathrm{e}}+\frac{R}{\rho} u_{\mathrm{a}}=0
$$

Cette condition peut être assurée par un ajustement de la déviation magnétique (modification de $u_{\mathrm{a}}$ ) ou par une légère correction de l'étalonnage du prisme électrostatique (action sur $u_{\mathrm{e}}$ ). Nous avons retenu le dernier procédé, plus facile à contrôler.

Ce réglage instrumental a reposé sur les émissions ${ }^{16} \mathrm{O}^{-}$et ${ }^{63} \mathrm{Cu}^{-}$mesurées sur le monocristal d'oxyde cuivreux.

Partant d'un réglage à $0,1 \mathrm{eV}$ près, tel que $\nu_{63}-\nu_{16}=0,1 \mathrm{~V}$ on obtient d'après la relation (14) $\nu_{63}-\nu_{16}=\nu_{16} \quad$ d'où $\quad \nu_{16}=-0,134 \mathrm{~V} \quad$ et $\nu_{63}=-0,034$.

La relation (14) fournit les autres écarts :

$\nu_{18}-\nu_{16}=0,015 \mathrm{~V} \quad \nu_{65}-\nu_{63}=1 \times 10^{-5} \mathrm{~V}$.

Ainsi, les quatre distributions examinées ont ainsi leurs origines confondues à $0,10 \mathrm{eV}$ près. Mais l'ajustage expérimental initial est sans doute plus précis que $0,1 \mathrm{eV}$.

Un calcul analogue montre que les différences relatives de masse entre les isotopes de l'oxygène et a fortiori du cuivre sont nettement trop petites par contre pour servir de base à un ajustage satisfaisant :

$\nu_{18}-\nu_{16}=0,1$ conduit à $\nu_{16}=-0,9$ et $\nu_{18}=-0,8$ mais comme $\nu_{63}-\nu_{16}=-0,67$ d'après (14) $\nu_{63}=-1,57$

Il serait pourtant préférable d'opérer ainsi puisque les différences de distribution énergétiques restent bien plus faibles entre isotopes qu'entre espèces ioniques en dépit des discriminations isotopiques intervenant pour l'émission (12).

\section{Bibliographie}

[1] Blandin A., Nourtier A. et Hone D. W., J. Phys. France 37 (1976) 369 ;

Lang N. D. et Norskov J. K., Phys. Scr. 6 (1983) 15.

[2] Blaise G. et Slodzian G., Surf. Sci. 40 (1973) 708.

[3] WittmaAcK K., Phys. Scr. 6 (1983) 71.

[4] Lingren S. A. et Wallden L., Solid State Commun. 25 (1978) 13.

[5] Bernheim M., Rebière J. et Slodzian G., J. Microsc. Spectrosc. Electr. Paris V5 (1980) 261 ;

Bernheim M. et Slodzian G., J. Microsc. Spectrosc. Electr. Paris 6 (1981) 141.

[6] Bernheim M. et Le Bourse F., Nucl. Instrum. Method. B27 (1987) 94.

[7] Bernheim M., Blaise G. et Slodzian G., Int. J. Mass. Spectrom. Ion Phys. 10 (1972) 293.
BERNHEIM M., Thèse, Orsay (1973).

[8] Slodzian G., Surf. Sci. 48 (1975) 161.

[9] De Bièvre P., Gallet M., Holden N. E. et Barnes J. L., J. Phys. Chem. Ref. Data 13 (1984) 809.

[10] Bernheim M. et Slodzian G., SIMS VI Conf. Eds. A. Benninghoven, A. Huber, H. W. Werner (Wiley) 13.3 (1988) p. 809.

[11] Kiejna A. et Wojciechowski K. F., Prog. Surf. Sci. 11 (1981) 293-338;

HÖlzl J., Schulte F. K., Springer Tracts Mod. Phys. 85 (Springer Verlag) 1979.

[12] Slodzian G., Phys. Scr. 6 (1983) 54.

[13] Hennequin J. F., Surf. Sci. (1988) 245.

BERNHEIM M. et SOYRIS N. (non publié). 